\title{
To Study the Antimicrobial Stewardship Program in a Large Tertiary Care Teaching Center
}

\author{
${ }^{1}$ Madhav Madhusudan Singh, ${ }^{2}$ Shakti Kumar Gupta, ${ }^{3}$ YK Gupta, ${ }^{4}$ DK Sharma, ${ }^{5}$ Aarti Kapil
}

\begin{abstract}
Introduction: As antimicrobial resistance continues to increase and new antimicrobial development stagnates, antimicrobial stewardship programs are being implemented worldwide. The goal of antimicrobial stewardship is to optimize antimicrobial therapy with maximal impact on subsequent development of resistance. Thirty to fifty percent of hospitalized patients receive antimicrobial therapy. Previous data suggest that inappropriate use results in higher mortality rates, longer lengths of stay, and increased medical costs. Antimicrobial stewardship programs (ASPs) reduce the improper use of antimicrobials and improve patient safety. Despite increased awareness about the benefits of these programs, few medical and surgical ASPs exist and fewer comprehensive studies evaluate their effects.
\end{abstract}

Aim: To study the antimicrobial stewardship program in a large tertiary care teaching center.

\section{Objectives}

- To study the antibiotic prescribing practices in a tertiary care government hospital

- To compare the antibiotic prescribing practices with the standard guidelines available with the hospital

- To make recommendation if any for rational use of antibiotics.

\section{Materials and methods}

- Review of literature

- Prospective study of 15 days in selected general medicine and general surgery ward in which 5 to 6 reading will be taken in to know the antibiotic prescribed to patients.

- Retrospective study of 15 days for study of patient records to know the antibiotic prescribed to patients.

\footnotetext{
${ }^{1}$ Joint Director [Medical Services (Trg) and Faculty], ${ }^{2,4}$ Medical Superintendent, ${ }^{3}$ Professor and Head, ${ }^{5}$ Professor

${ }^{1}$ Department of PSM, Army College of Medical Sciences, New Delhi, India

${ }^{2}$ Department of Hospital Administration, RPCOS; All India Institute of Medical Sciences, New Delhi, India

${ }^{3}$ Department of Pharmacology, All India Institute of Medical Sciences, New Delhi, India

${ }^{4}$ Department of Hospital Administration, All India Institute of Medical Sciences, New Delhi, India

${ }^{5}$ Department of Microbiology, All India Institute of Medical Sciences, New Delhi, India

Corresponding Author: Madhav Madhusudan Singh, Joint Director [Medical Services (Trg) and Faculty], Room No. 96 O/O DGMS (Army), Ministry of Defence, New Delhi, India e-mail: mmsingh2011@gmail.com
}

- Interaction with faculty and senior residents of general medicine and surgery to know about the pattern of infection and antibiotic prescription.

- Interaction with microbiology department and their faculty to know the microbial resistance pattern and possible suggestion which need to be incorporated in antibiotic Stewardship program.

Results: The present study on antibiotic prescribing practices was undertaken in a super specialty hospital at New Delhi. A sample size of 100 case records was considered. There is no such stewardship program in tertiary care hospital, although it was demanded in various forum and meetings. There are no recommendations available either for patients of renal failure or other such compromised metabolic or immune states in the form of written antibiotic stewardship program of the hospital. The appropriateness of antibiotics prescribed in the case records was examined in light of the antibiotic stewardship program of the hospital. It was found that the overall adherence to antibiotic stewardship program was nil as no existing antibiotic stewardship program is exiting in this hospital. Gautum Dey in a study conducted at this hospital in New Delhi found that in $40.7 \%$ preoperative cases and $60.3 \%$ postoperative cases two or more than two antibiotics were given. The author has also commented that there was no evidence of adhering to antibiotic stewardship program or utilising culture and sensitivity reports to guide the therapy. The data obtained from the present study on further analysis has shown that in seven cases, the antibiotics prescribed were inadequate in terms of dose and duration. Thus resulting in an apparently lower cost of treatment than what was recommended by the antibiotic stewardship program of the hospital. Although such inappropriate prescription results in increased chances of antibiotic resistance, the immediate or short-term effects are not very conclusive. It is observed that there were $26(26 \%)$ cases in medical and $12(12 \%)$ cases in surgery disciplines in which the initial and final diagnosis was different. Uncertainty about the final diagnosis promotes empirical prescribing practices.

Conclusion: Antimicrobial stewards are a prominent part of local and national efforts to contain and reverse antimicrobial resistance. A range of intervention options is available with varying levels of resources and can yield substantial improvements in morbidity, mortality, quality of care, and cost. The cost of delivering such programs is dwarfed by the benefits and provides an opportunity for hospital epidemiologists to garner support. This suggests that antimicrobial management programs belong to the rarefied group of truly cost saving quality improvement initiatives. Considering the enormous implications of antibiotic resistance, it is necessary that we act in haste, lest our wonder drugs and magic bullets become ineffectual. Future systems promise greater integration and analysis of data, facilitated delivery of information to the clinician, and rapid and expert decision support that will optimize patient 
outcomes while minimizing antimicrobial resistance. They may also offer our best hope for avoiding an 'Antibiotic armageddon'. In addition, the ASP plays an integral role in providing guidance to clinicians and ensures that the appropriate antimicrobial agents are used.

Keywords: Antibiotic, Stewardship program, Antimicrobial resistance, Rational use.

How to cite this article: Singh MM, Gupta SK, Gupta YK, Sharma DK, Kapil A. To Study the Antimicrobial Stewardship Program in a Large Tertiary Care Teaching Center. Int J Res Foundation Hosp Healthc Adm 2015;3(1):13-24.

\section{Source of support: Nil}

\section{Conflict of interest: None}

\section{INTRODUCTION}

The majority of the hospitalized patients receive antibiotics for therapy or prophylaxis during their inpatient stay. It has been estimated that at least $55 \%$ of patients receive antibiotics needlessly. Reasons include inappropriate prescribing for antibiotic prophylaxis, continuation of empiric therapy despite negative cultures in a stable patient, and a lack of awareness of susceptibility patterns of common pathogens.

Over prescribing not only increases the costs of healthcare, but may result in super infection due to antibiotic resistant bacteria, as well as opportunistic fungi, and may increase the likelihood of an adverse drug reaction. On the other hand, not prescribing (when there is an urgent need at the bedside) may also lead to serious consequences.

Up to $30 \%$ of hospital patients receive antimicrobial agents, and expenditure for these drugs may comprise 10 to $40 \%$ of the hospital pharmacy budget.

Antimicrobial resistance (AMR) has emerged as a major public health problem all over the world. Infections caused by resistant microbes fail to respond to treatment, resulting in prolonged illness and greater risk of death. Treatment failures also lead to longer periods of infectivity, with increased numbers of infected people moving in the community. This in turn exposes the general population to the risk of contracting a resistant strain of microorganisms. When these become resistant to first-line antimicrobials, the prohibitive high cost of the second-line drugs may result in failure to treat these diseases in many individuals. Most alarming of all are the diseases caused by multidrug-resistant microbes, which are virtually nontreatable, and thereby create a 'postantibiotic era' scenario.

The rational use of drugs advocates that patients receive medicines appropriate to their clinical needs, in doses that meet their own individual requirements, for an adequate period of time, and at the lowest cost to them and the community. ${ }^{1,2}$ Standardized prophylaxis/ therapy is necessary not only to achieve optimum patient care but also to retard the emergence of drug resistance, bring down the cost of medical care and enable effective utilization of resources. ${ }^{3}$

The irrational use of drugs including improper indication, dosage, duration, combination and associated instruction results in increased incidence of resistance to commonly used antibiotics and increased expenditure on healthcare. It is estimated that almost one-third of the hospitalized patients receive antibiotics, half of which is unnecessary. ${ }^{4}$

In view of the above, it is necessary that healthcare institutions should have a stewardship program/guidelines according to which antibiotics should be prescribed. Antibiotic stewardship program is a set of guidelines laid down to optimize the use of antibiotics in many healthcare institutions. The proper use of such stewardship program helps in decreasing antibiotic resistance and to provide maximum benefit to the patients both in terms of clinical as well as economic outcome. The antibiotic stewardship program should take into consideration various clinical, diagnostic, bacteriological and pharmacological criteria.

It is observed though that the guidelines for the use of antibiotics/antibiotic stewardship program exist in many hospitals but the same are not implemented strictly. The cost incurred in inappropriate antibiotic therapy not only adds to the cost of healthcare expenditure but also adds to the disease burden by increasing the incidence of resistance to antibiotics among bacteria. ${ }^{6,7}$ This aspect can only be ascertained with the help of an evidence based study.

The global spread of bacteria carrying the New Delhi metallo- $\beta$-lactamase-1 (NDM-1) enzyme through India, Pakistan, and the United Kingdom-and now half a dozen other countries-has sparked much media coverage. The outbreak's importance stems from the broad resistance to all antibiotics except tigecycline and colistin seen in bacterial strains carrying the gene for NDM-1 and from the ready transmission across borders.

Sharing of expertise, cooperation, and collaboration between the clinicians using antibiotic therapy and the clinical microbiologists at the regional levels may be simplest and most useful public health measure to optimize the use of antibiotics and manage infectious diseases.

A master antibiogram for a region would allow a tertiary care institution to consider resistance patterns in hospitals referring patients and to select appropriate antimicrobial therapy or change drugs in nonresponding patients. Antimicrobial resistance data generated by this approach will have better day to day application. 
The concept of 'empirical antimicrobial therapy' would then be changed to that of 'presumptive antimicrobial therapy' based on common pathogens, known susceptibility pattern and host factors in any given region. It is necessary to define an antimicrobial stewardship program as ongoing effort by a healthcare institution to optimize antimicrobial use among hospitalized patients to improve patient outcomes, ensure cost effective therapy and reduce adverse sequelae of antimicrobial use.

Antimicrobial stewardship lies at the intersection of infectious disease. Infection control, safety and quality improvement, and cost containment. It has been defined as the 'appropriate selection dosing, and duration of antimicrobial therapy to achieve optimal efficacy in managing infections. It has been, thus, an important tool in the effort to reduce inappropriate use of antimicrobials and subsequent development of both resistant microorganisms and drug related adverse events.

Effective antimicrobial stewardship requires the collaboration of many disciplines, including medical, surgical services, nursing, infection control, laboratory, and pharmacy. Monitoring antimicrobial use is an important component of stewardship because of the close relationship between use of antimicrobial agents and the emergence of bacterial resistance. For example, excessive use of 3rd generation cephalosporin and of vancomycine has been linked to emergence of several resistant organisms, including MRSA, VRE and ESBL producing E. coli and Klebsiella spp. ${ }^{1}$

In view of the above present study was undertaken in a tertiary care government hospital at New Delhi to study and analyze the antibiotic prescribing practices of doctors in relation to its antibiotic stewardship program, duly considering the concepts of evidence based medicine.

\section{NEED OF THE STUDY}

- To ensure that antibiotics are used properly

- To reduce the overuse/misuse of antibiotics

- To reduce the development of drug resistance

- To reduce the side effect of the drugs

- To reduce the overall cost of treatment

- To reduce the morbidity and mortality

- To promote evidence based medicine

- To improve patient care by promoting the best practice in antibiotic prophylaxis and therapy, and make better use of resources by using cheaper drugs wherever possible.

- To retard the emergence and spread of multiple antibiotic resistance bacteria by improving education of the related doctors by providing guidelines for appropriate therapy.

\section{Exclusion Criteria}

- Specific instruction regarding difficult to treat organisms or infections are not included within the scope of this study

- Antibiotic for surgical prophylaxis, urinary tract infection prophylaxis, post-splenectomy prophylaxis or any other form of prophylaxis

- Antibiotics for use as a motility stimulants

- Any topical antimicrobial applied to skin, eye, ear, nasal passages or genital area for local effects

- Antiretroviral, antiprotozoal, or anthelmintic preparations.

\section{MATERIALS AND METHODS}

- Review of literature

- Prospective study of 15 days in selected general medicine and surgery ward in which five to six reading will be taken into know the antibiotic prescribed to patients as format sown as Appendix $\mathrm{B}$ and $\mathrm{C}$.

- Retrospective study of 15 days for study of patient records to know the antibiotic prescribed to patients as format shown as Appendix A and D.

- Interaction with faculty and senior residents of general medicine and surgery: to know about the pattern of infection and antibiotic prescription.

- Interaction with microbiology department and their faculty to know the microbial resistance pattern and possible suggestion which need to be incorporated in antibiotic stewardship program.

\section{RESULTS AND OBSERVATIONS}

1. The study was conducted in a super specialty tertiary care government hospital at New Delhi. The hospital is also a teaching center for undergraduates, postgraduates, nurses, and paramedics. There are total 2,178 beds with 21 wards in this hospital having an average bed occupancy rate of more than $79.9 \%$. The average length of hospital stay is 5.9 days while net death rate is $2.7 \%$. The crude infection rate is $7.60 \%$. The hospital has an average of 2,93,086 admissions per year and approximately 25,14,854 outpatient attendance.

2. The data collection in the study was conducted over a period of 3 months from May to July 2011. For the purpose of the study, the case sheets of in-patients admitted in the acute care areas of the hospital were studied. These case sheets were obtained from the medical records department of the tertiary care hospital. The case sheets of discharged patients having antibiotic prescriptions initiated by the treating doctors were considered as a study population to draw the sample. 
Appendix A

Retrospective study of patient in general medicine ward

Name
Age
Sex
Adm no.
Specialty
Date of adm.
Date of discharge
Ward/bed no.
Initial diagnosis
Final diagnosis
Details of antibiotic

Criteria for prescribing antibiotic

As per antibiotic stewardship program or not

Instruction entered in notes or not

Appendix B

Prospective study of patient in general medicine ward

Name
Age
Sex
Adm no.
Specialty
Date of adm.
Date of discharge
Ward/bed no.
Initial diagnosis
Final diagnosis

Criteria for prescribing antibiotic

As per antibiotic stewardship program or not

Instruction entered in notes or not

3. A total of 100 such case records, obtained by systematic random sampling, were studied and the following information was obtained from it and compiled in an MS Excel data sheet and later analyzed with the help of SPSS:

- Admission and discharge number of the patient

- Final diagnosis

- Initial diagnosis

- Sex

- Specialty

- The antibiotics given-dose and duration

- Criteria used for prescribing antibiotics
- Whether the prescription was as per the antibiotic stewardship program of the hospital

- Instructions/notes entered in the case records as regards the antibiotic administration.

4. Study of antibiotic stewardship program of the hospital as follows:

- The antibiotic stewardship program is not existing in this hospital although hospital infection control program is there for which there is a hospital infection control program and committee.

- No documentary evidence was available that the senior specialists were consulted prior to starting 
Appendix C

Prospective study of patient in general surgery ward: C7/D7

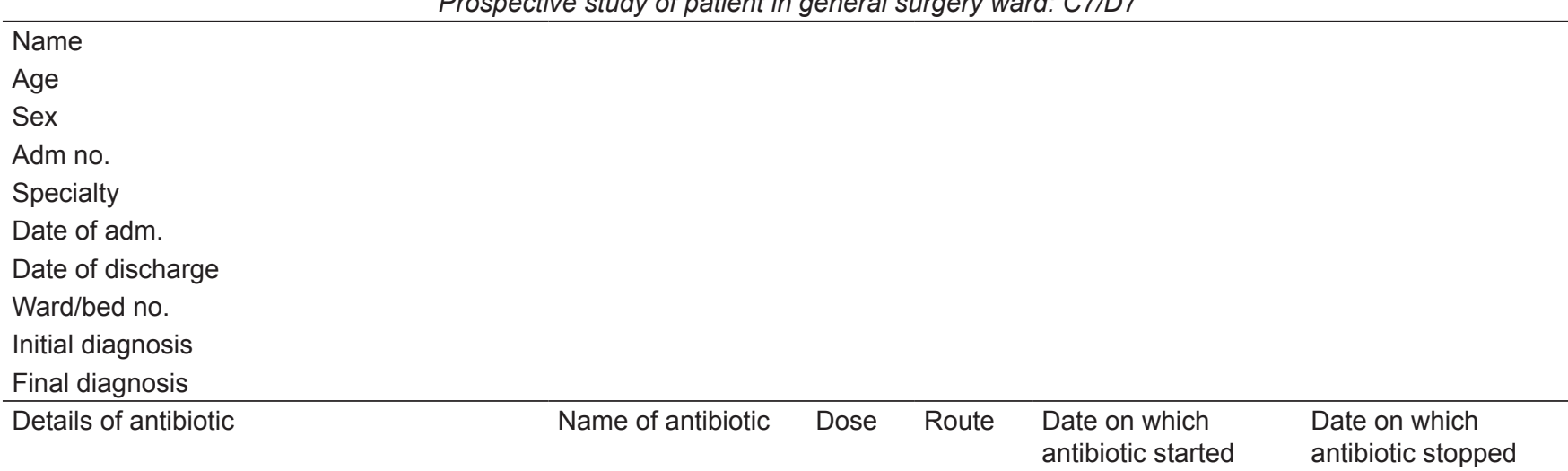

Criteria for prescribing antibiotic

As per antibiotic stewardship program or not

Instruction entered in notes or not

Appendix D

Retrospective study of patient in general surgery ward

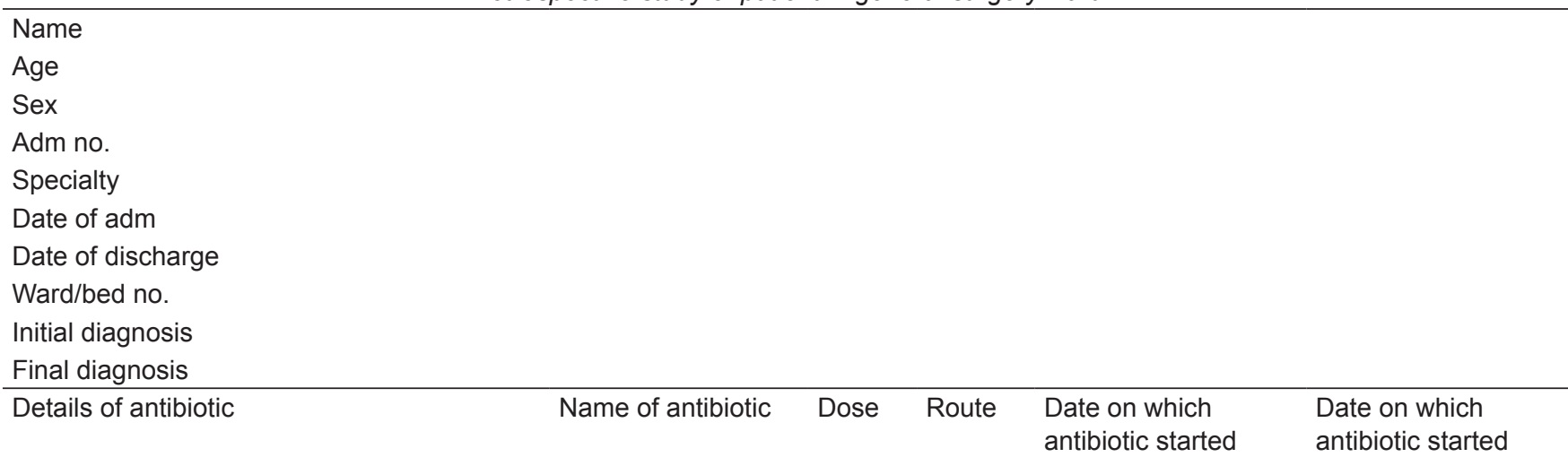

Criteria for prescribing antibiotic

As per antibiotic stewardship program or not

Instruction entered in notes or not

higher and newer antibiotics and in case of treatment failure.

5. Demographic profile of the sample population: The demographic profile of the sample population was studied as per the age, sex and category (Civilian, EHS patient) as follows:

- Sex: The genderwise distribution of case records is shown in Table 1 and Figure 1.

- Age: the agewise distribution of the case records is as shown in Table 2. The mean age of the sample population was 47.7 with a standard deviation of 17.09884207. Figure 2 shows the distribution of cases as per various age groups. The maximum number of cases are in age group of 61 to 70 years (21\%), followed

Table 1: Genderwise distribution of sample population

\begin{tabular}{lll}
\hline Sex & Number of case records & Percentage \\
\hline Male & 62 & 62 \\
Female & 38 & 38 \\
\hline Total & 100 & 100 \\
\hline
\end{tabular}




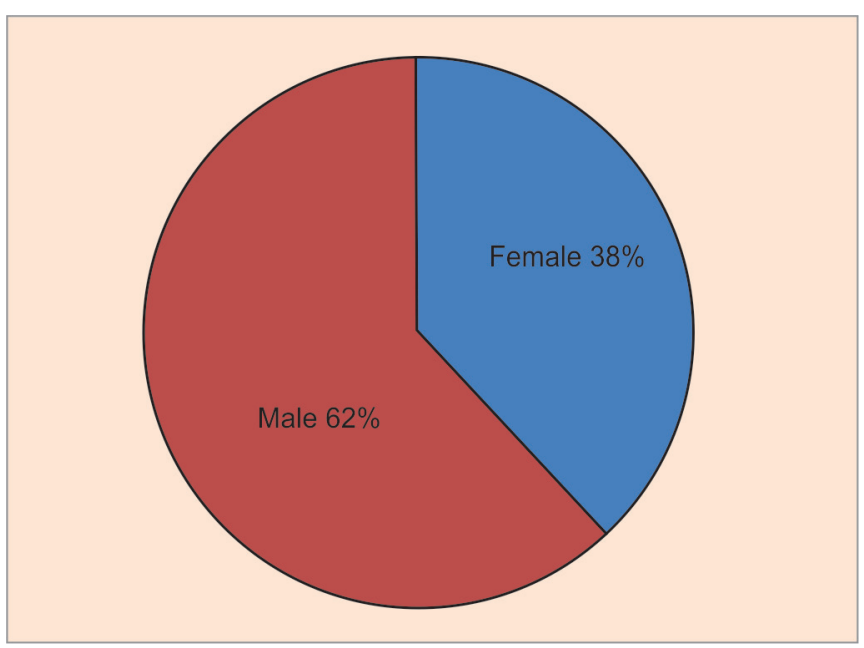

Fig. 1: Male female ratio of entire sample

by the age group of 31 to 40 years (20\%) (Table 3). The maximum age of patient was recorded 82 while minimum was 13 years.

Age distribution of female sample population: Table 4 illustrates the age distribution of sample cases among the female patients. Table 4 shows that mean age of the female population was 40.43 years with a standard deviation of 16.4351 .

Age distribution of male sample population: Table 5 illustrates the age distribution of sample cases among the male patients. Table 5 shows that mean age of the male population was 47.35 years with a standard deviation of 17.00 .

Table 2: Mean age of sample population

\begin{tabular}{lllll}
\hline Observation & Mean & Std. dev. & Mode & Median \\
\hline 100 & 47.7 & 17.09 & 70 & 47.5 \\
\hline
\end{tabular}

Table 3: Mean age of sample population

\begin{tabular}{lll}
\hline Age group & Number of cases & Percentage \\
\hline $0-10$ & Nil & 0 \\
$11-20$ & 6 & 6 \\
$21-30$ & 11 & 11 \\
$31-40$ & 20 & 20 \\
$41-50$ & 16 & 16 \\
$51-60$ & 19 & 19 \\
$61-70$ & 21 & 21 \\
$>70$ years & 7 & 7 \\
\hline
\end{tabular}

Table 4: Mean age of female case record

\begin{tabular}{lllllll}
\hline $\begin{array}{l}\text { Female } \\
\text { observations }\end{array}$ & $\begin{array}{l}\text { Total } \\
\text { sample }\end{array}$ & Mean & $\begin{array}{l}\text { Std. } \\
\text { dev. }\end{array}$ & $\begin{array}{l}\text { Vari- } \\
\text { ance }\end{array}$ & Mode & Median \\
\hline 38 & 100 & 40.43 & 16.43 & 317.84 & 70 & 43.0 \\
\hline
\end{tabular}

Table 5: Mean age of male case record

\begin{tabular}{lllllll}
\hline $\begin{array}{l}\text { Male } \\
\text { obser- } \\
\text { vations }\end{array}$ & $\begin{array}{l}\text { Total } \\
\text { sample }\end{array}$ & Mean & $\begin{array}{l}\text { Std. } \\
\text { dev. }\end{array}$ & Variance & Mode & Median \\
\hline 62 & 100 & 47.35 & 17.0057 & 286.983 & 61 & 52 \\
\hline
\end{tabular}

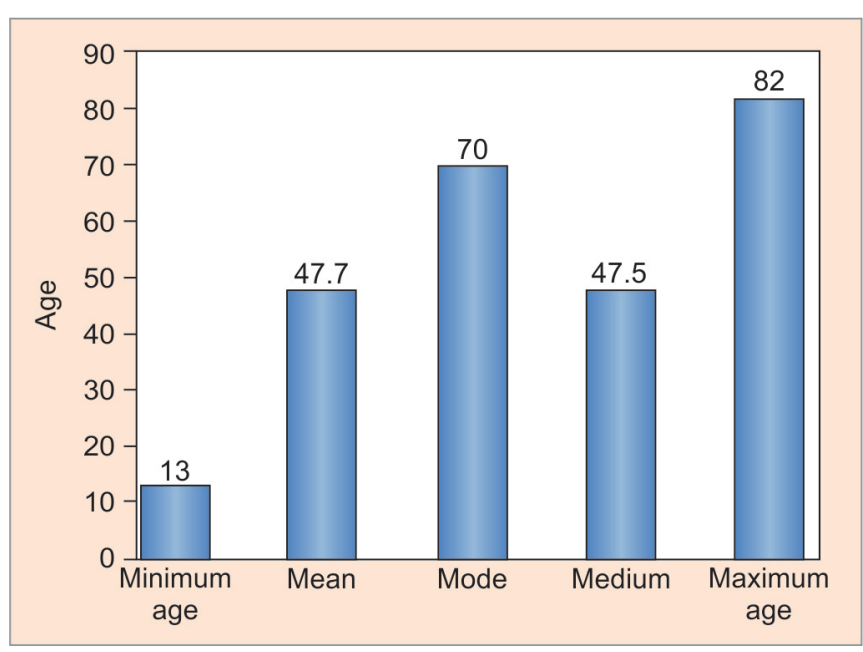

Fig. 2: Age distribution of entire sample

\section{Diagnosis Commonly encountered in the Sample Population}

During the course of the study following diseases were commonly encountered under the broad disciplines.

- Surgical case records: Table 6 shows the diseases commonly encountered among the surgical cases in the study along with their frequency distribution.

- Medical case records: Table 7 shows the diagnosis commonly encountered among the medical cases.

- Final diagnosis not matching with the initial diagnosis: In a large number of cases, the final diagnosis

Table 6: Common surgical diagnosis among the sample records

\begin{tabular}{|c|c|c|}
\hline Diagnosis & Frequency & $\begin{array}{l}\text { Percentage (out of total } \\
\text { sample case including } \\
\text { medicine cases) }\end{array}$ \\
\hline $\begin{array}{l}\text { Abdominal Koch's with } \\
\text { Rt hemicolectomy }\end{array}$ & III & 3 \\
\hline Abscesses & III & 3 \\
\hline Adrenal mass & I & 1 \\
\hline Appendicitis & III & 3 \\
\hline $\begin{array}{l}\text { Biliary stricture/sepsis/ } \\
\text { carcinoma }\end{array}$ & IIIII & 5 \\
\hline Ca. breast & III & 3 \\
\hline Ca gallbladder & 1 & 1 \\
\hline Ca. caecum/rectum & IIIII & 5 \\
\hline $\begin{array}{l}\text { Cholecystitis (acute and } \\
\text { chronic) }\end{array}$ & IIIII, III & 8 \\
\hline Dentigerous cyst & 1 & 1 \\
\hline Fistula & II & 2 \\
\hline $\begin{array}{l}\text { Gastrointestinal stromal } \\
\text { tumor }\end{array}$ & I & 1 \\
\hline Inguinal hernia & III & 3 \\
\hline Orchitis & II & 2 \\
\hline Perforation peritonitis & II & 2 \\
\hline Sepsis & III & 3 \\
\hline Sigmoid volvulus & II & 2 \\
\hline Testicular tumor & II & 1 \\
\hline Traumatic pancreatitis & 1 & 1 \\
\hline
\end{tabular}


Table 7: Common medical diagnosis among the sample records

\begin{tabular}{|c|c|c|}
\hline Diagnosis & Frequency & $\begin{array}{l}\text { Percentage (out of total sample case } \\
\text { including surgical cases) }\end{array}$ \\
\hline Pneumonia & IIIII & 5 \\
\hline Upper respiratory tract infection & I & 1 \\
\hline Fever (inv) & IIIII & 5 \\
\hline Malaria & I & 1 \\
\hline Exudative pleural effusion & IIIII & 4 \\
\hline Pancytopenia & I & 1 \\
\hline Type II respiratory failure & IIII & 4 \\
\hline Asthma & IIIII & 5 \\
\hline Koch's disease & III & 1 \\
\hline Obstructive pulmonary disease & IIIII, IIIII & 7 \\
\hline Seizures & I & 1 \\
\hline Septic shock & II & 2 \\
\hline Severe sepsis & II & 2 \\
\hline Hip replacement with infection & I & 1 \\
\hline Tuberculosis meningitis with septic shock encephalitis & I & 1 \\
\hline Diabetes mellitus, hypertension, multiple myeloma and URTI & I & 1 \\
\hline Diabetes mellitus, hypertension and tuberculosis & I & 1 \\
\hline Oraganophosphorus poisoning & I & 1 \\
\hline Diabetes mellitus, hypertension and severe sepsis & I & 1 \\
\hline Non-insulin dependent diabetes mellitus and IDL & II & 2 \\
\hline Tubercular meningitis & I & 1 \\
\hline Cystic kidney disease and sepsis & I & 1 \\
\hline Hypothyroidism and amenorrhea & I & 1 \\
\hline
\end{tabular}

Table 8: Surgical case records with different initial and final diagnosis

\begin{tabular}{llll}
\hline Final diagnosis & Initial diagnosis & Frequency & Percentage (out of 50 surgery case) \\
\hline Acute appendicitis & Pain abdomen (inv) & 3 & 6 \\
Acute cholecystitis & Pain abdomen & 2 & 4 \\
Symptomatic cholecystitis & Pain abdomen & 1 & 2 \\
\hline Total & & 12 \\
\hline
\end{tabular}

was not matching with the initial diagnosis as given below:

- Surgical case records with different initial and final diagnosis: The surgical case records in which the final diagnosis did not match with initial diagnosis are shown in Table 8.

- Medical case records with different initial diagnosis and final diagnosis: The medical cases records in which the initial and final diagnoses were different are shown in the Table 9.

- Comparison of the antibiotics prescribed in the case sheets in the light of antibiotic policy:

- The case records selected through a systematic random sampling method were studied in respect of the various antibiotics prescribed. The antibiotics prescribed were analyzed according to the correctness of dose and duration as recommended by the antibiotic policy of the other hospitals and standard textbooks. Wherever, there was an ambiguity in the selection, duration, dose or combination
Table 9: Medical case records with different initial and final diagnosis

\begin{tabular}{llll}
\hline & & & $\begin{array}{l}\text { Percentage } \\
\text { (out of 50 }\end{array}$ \\
Final diagnosis & Initial diagnosis & Frequency & medical case) \\
\hline $\begin{array}{l}\text { Bronchitis } \\
\begin{array}{l}\text { Upper respiratory } \\
\text { tract infection }\end{array}\end{array}$ & Fever (inv) & 3 & 6 \\
$\begin{array}{l}\text { Obstructive } \\
\text { pulmonary } \\
\text { disease }\end{array}$ & 1 & 2 \\
$\begin{array}{l}\text { Septic shock } \\
\begin{array}{l}\text { Tubercular } \\
\text { meningitis }\end{array}\end{array}$ & Giddiness & 2 & 6 \\
$\begin{array}{l}\text { Cystic kidney } \\
\text { disease and } \\
\text { sepsis }\end{array}$ & UTI & 1 & 2 \\
$\begin{array}{l}\text { Hypothyroidism } \\
\text { and amenorrhea } \\
\text { OP poisoning }\end{array}$ & Weakness & 1 & 2 \\
\hline Total & Unknown & 1 & 2 \\
\hline
\end{tabular}


of therapy the concerned senior specialists were consulted.

- Many a times (seven out of 100), it was found that the patients were prescribed less duration of antibiotics as compared to that recommended by the antibiotic policy. Such cases which result in an apparently lower cost of treatment, but may lead to long-term effects of bacterial resistance in the individual spreading later to the community, have been excluded from the final calculation regarding cost implication of not adhering to the antibiotic policy of the hospital.

\section{DISCUSSION AND CONCLUSION}

1. The present study on antibiotic prescribing practices was undertaken in a super specialty hospital at New Delhi. A sample size of 100 case records was considered. The observations made on antibiotic stewardship program and on the records of the hospital are discussed in succeeding paragraphs in accordance with objectives of the study.

2. Antibiotic stewardship program of the hospital:

- There is no such stewardship program in this hospital, although it was demanded in various forum and meetings.

Mol et $\mathrm{al}^{2}$ in a time series intervention analysis, carried out at a University teaching hospital in Groningen, Netherlands, showed that updating the antimicrobial guidelines in close collaboration with the specialists and distributing it in a paperback and electronic format improved the prescribing compliance by $15.5 \%$.

The antibiotic stewardship program of the Calderdale and Huddersfield Hospital, under NHS Trust, ${ }^{3}$ deals in great detail with the surgical antimicrobial prophylaxis, its timings and the details of drugs given in various kinds of surgeries.

- There are no recommendations available either for patients of renal failure or other such compromised metabolic or immune states in the form of written antibiotic stewardship program of the hospital. However, in many countries, the antibiotic stewardship program also includes the recommendations and precautions to be observed while prescribing for renal failure and other miscellaneous diseases. ${ }^{4}$

- Many hospitals in other countries display their antibiotic policies on their website. One may also download it on request. Christian Medical College, Vellore, ${ }^{5}$ has published its antibiotic stewardship program in the form of a small booklet, which is laminated and easily fits inside a pocket. This allows the antibiotic stewardship program to be carried easily by the junior residents and is, thus, easily accessible (copy enclosed with the document as enclosure 2). The stewardship program has incorporated a number of flowcharts and clinical guidelines which allows easy referral. Wiffen and White ${ }^{6}$ in a study conducted over 41 hospitals under trust of NHS, found that the policies provided had a wide range of presentation styles ranging from an interactive computer program to a single-folded card. The most popular formats were A4 (11 policies), pocket size (12 policies) or A5 (11 policies).

3. The appropriateness of antibiotics prescribed in the case records was examined in light of the antibiotic stewardship program of the hospital. It was found that the overall adherence to antibiotic stewardship program was nil as no existing antibiotic stewardship program is exiting in this hospital.

Studies by authors in other countries have shown a much higher prevalence of adherence to antibiotic stewardship program. Ozkurt et al, ${ }^{7}$ in a study conducted in a Research Hospital of the Atakurk University Medical School in Turkey, showed an overall prevalence of adherence to antibiotic stewardship program as $47.3 \%$.

4. Fijn et $\mathrm{al}^{8}$ in a multicentric retrospective explorative cohort study found that, in $24.3 \%$ cases, the antibiotic prescribed was not indicated and, in $55.2 \%$ cases, the antibiotic prescribed was not the first drug of choice. In a prospective, multicenter audit of elective procedures, carried out in 13 Dutch hospitals with a sample size of 1,763 , by Van Kasteren et $\mathrm{al}^{9}$ the overall adherence of antibiotic guidelines was found to be $28 \%$. It was seen that antibiotic choice, duration, dose, dosing interval and timing of the first dose were concordant with the hospital guideline in $92,82,89,43$ and $50 \%$ cases respectively.

In the study conducted by Ozkurt, ${ }^{7}$ it was found that appropriateness of antibiotics was 45.3 and $21.7 \%$ in surgical and medical wards respectively. Dey, ${ }^{10}$ in a study conducted at AIIMS, New Delhi, found that in 40.7\% preoperative cases and $60.3 \%$ postoperative cases two or more than two antibiotics were given. The author has also commented that there was no evidence of adhering to antibiotic stewardship program or utilising culture and sensitivity reports to guide the therapy.

5. The data obtained from the present study on further analysis has shown that in seven cases, the antibiotics prescribed were inadequate in terms of dose and duration. Thus, resulting in an apparently lower cost of treatment than what was recommended by the antibiotic stewardship program of the hospital. Although such inappropriate prescription results in increased 
chances of antibiotic resistance, but the immediate or short-term effects are not very conclusive.

6. It is observed from Tables 8 and 9, that there were 26 (26\%) cases in medical and $12(12 \%)$ cases in surgery disciplines in which the initial and final diagnosis was different. Uncertainty about the final diagnosis promotes empirical prescribing practices.

\section{RECOMMENDATIONS}

1. The study on antibiotic prescribing practices in a tertiary care hospital was undertaken by studying the case records of 100 inpatients admitted to the acute wards (medicine and surgery) of the hospital. Keeping in view the observations made during the study, certain recommendations are offered on appropriate prescription of antibiotics. The same are presented in succeeding paragraphs.

2. Antibiotic stewardship program: The following recommendations are proposed for the antibiotic stewardship program of the hospital:

- The aim of antibiotic stewardship program of the hospital should be promotion of rational prescribing practices and prevent emergence of new and reduction of existing resistant strains of pathogens.

- The stewardship program should be reviewed six monthly on the basis of the microorganism sensitivity pattern. The clinicians pertaining to major disciplines must be an integral part of committee formulating the antibiotic stewardship program.

- The stewardship program should be as comprehensive as possible and nothing should be left to imagination as it is used by the senior most to the most junior doctors of the hospital.

- The stewardship program must cover all the common diseases and also important uncommon conditions for different age groups.

- The stewardship program should include the dose, duration and schedule of antibiotics for an average adult and also mention the per $\mathrm{kg}$ dose of the medicine.

- It is recommended that the latest drug of choice specific to the micro-organisms on the basis of the sensitivity pattern pertaining to a particular diagnosis should be mentioned than on the basis of the type of cases, like OPD/IPD or major/minor case, etc.

- The stewardship program should also indicate if the choice of antibiotics differs in case of OPD patients for the same diagnosis. However, as far as possible, the clinical and more preferably the bacteriological reports to be kept in mind prior to any prescription of antibiotics.

- The stewardship program should include associated instructions as following:

- For the clinicians: In terms of dose, duration, sample/repeat sample with intervals to be sent, interactions with other drugs or combinations of drugs to be used and precautions to be used in case of compromised metabolic/immunocompromised status of the patient.

- For the patients: These instructions should be conveyed by hospital staff to the patients and should be pertaining to the timing of the dose, duration, relation with meals, side effects and interaction with other drugs.

- For the nurses: In addition to the relevant information given at serial (i) above, instructions to include any side effects which is to be monitored.

- The antibiotic stewardship program of the hospital should be easily accessible preferably on its website. It should also be made available in the form of a small booklet to all concerned.

- An effective program of antibiotic reform requires a team approach. This starts with formation of an antibiotic utilisation committee, chaired by a member of the infectious disease (medicine). There should also be representatives from pharmacy, microbiology, and infection control; depending on the size and structure of the hospital, representation from several other departments may be helpful (Table 10).

Direct involvement of a key administrator provides liaison to higher levels of hospital leadership, as well

Table 10: Composition of an antimicrobial stewardship committee

\begin{tabular}{l}
\hline Infectious disease Head (medicine): Chairman \\
Faculty incharge pharmacy \\
Head of microbiology \\
Head of pharmacology \\
Infection control practitioners \\
Head of surgery \\
Head of pediatrics \\
Head of gynecology \\
Head of ENT \\
Head of Eye \\
Intensivists \\
Head of emergency medicine \\
Member from hospital administration (preferably Medical \\
Superintendent) \\
Quality control manager \\
Data manager \\
Staff nurse
\end{tabular}


as vital support when reform efforts must be promoted among the medical staff. In addition, collaboration with quality improvement team may bring substantial administrative resources to bear and facilitates innovation and change. A small working group, including the chairman and pharmacy in charge with data manager, provides most of the effort, with advice from the larger committee.

Following components of antimicrobial stewardship program are as below:

- Data collection and target identification

- Formulary revision

- Microbiology testing and reporting

- Education

- Restriction policies

- Ordering policies

- Drug administration

- Limiting contact with pharmaceutical representatives. There are some Caveats when any hospital implements an antimicrobial stewardship program as shown in Table 11.

For maintaining and evaluating the success of program, it is essential to make administrators aware of significance of antimicrobial stewardship efforts and to periodically reinforce the importance of these endeavors. In addition to pharmacy budget savings, the volume and scope of interventions can be tallied, as well as rates of medication errors and adverse events. Antimicrobial usages and resistance patterns should be monitored to document successes and to provide alerts to new problems. Costs associated with specific diagnosis related groups may drop if targeted programs result in shorter length of hospitalization. These data should be shared with hospital administrators to reinforce the financial and clinical value of the program.

Benefits can be further expressed in measures of prevented morbidity, mortality, resistance, errors, adverse events, wasted medications and excess length of hospital stay, as well as the 'added value' of decreased adverse publicity and liability. Estimates of savings associated with these parameters may be based on published data if cost measuring databases are unavailable.

3. Prescribing practices: The supportive investigative results should be made available as early as possible

Table 11: Caveats when implementing an antimicrobial stewardship program

- Provide antimicrobial agent stewardship; do not become a policeman or zealot

- Avoid formulary changes for only short-term gain

- Financial concerns should not be more important than clinical efficacy and safety

- Formulary changes may necessitate changes in automated susceptibility testing in the hospitalised patients if technically feasible. This will enable the clinicians to reach a conclusive diagnosis earlier and to start treatment based on bacteriological or investigative results rather than empirical therapy. The administrator and senior specialists should stress upon all the clinicians to endorse the necessary associated instructions and prescribe legibly duly endorsing their signatures and stamps wherever necessary. Always send samples for microbiological workup before starting or changing antibiotics. There should be differentiation between contamination, colonisation and infection is important to prevent overuse of antibiotics. De-escalation of antibiotics should be done on the basis of clinical condition and microbiology report, for judicious use of antibiotics the appropriateness, efficacy, dosage and side effects must be reviewed on the daily basis. Shift to oral antibiotics from parenteral antibiotics as early as possible. It should be known to all that there is no substitute of hand washing in prevention of spread of nosocomial infections.

4. Sensitising the undergraduate students and postgraduate students to antibiotic stewardship program: It is recommended that undergraduate and postgraduate medical students must be sensitised about the concepts of antibiotic stewardship program and importance of maintaining a discipline in prescribing practices as they are at the most impressionable age.

5. Continuing medical education: It is strongly recommended that a continuing medical education program should be developed by the hospital incorporating various departments on regular basis to promote the adherence to antibiotic stewardship program. More stringent efforts should be made for adherence to the stewardship program by medical and surgical division as their compliance to adherence was minimum and they are supposedly the maximum users.

The hospitals and healthcare institutions should try to organise conferences and seminars wherein the latest surveillance studies and evidence-based treatment of various diseases/conditions may be discussed and subsequently incorporated in the antibiotic stewardship program. The hospital pharmacists and administrators may give data and feedback on prescribing behavior without any intention of fault finding or pin pointing any particular person or specialty.

6. Surveillance studies: There should be surveillance procedure to monitor the rate of healthcare associates infections. Following steps are recommended for surveillance procedure:

- Constitution of an antibiotic use committee in the hospital which should participate in surveillance activities. 
- Antibiotic susceptibility testing of appropriate microorganism.

- Antimicrobial testing for selected resistant isolates.

- Monitor the trends in prevalence of bacterial resistance to antimicrobial agents.

- To notify Hospital Infection Control Committee, if there is any unusual resistance pattern.

7. Monitoring antibiotic use: It is recommended that antibiotic use of the hospital should be monitored by the medical stores/pharmacy department. It should be reported to a committee responsible for appropriate utilization of medicines.

8. Weekly Bulletins should be circulated to all wards and regarding the availability of antibiotic in the stores.

9. Audit of antibiotic prescribing behavior: It is recommended that periodic audit should be undertaken to examine the appropriateness of antimicrobial use. The audit committee should comprise of clinicians from all major discipline among others and the committee should analyze following aspects:

- Use of antimicrobials as per the guidelines.

- Resistance pattern of pathogens in consultation with microbiology department.

- Reasons for poor outcome of patients if any.

10. Liaison and collaboration with local and regional healthcare institutions and laboratories: The hospitals and laboratories of a region should be organized in the form of a body wherein data about surveillance studies, resistance patterns, and antibiotic use can be shared between the members of the group. The antibiotic stewardship program of all hospitals/healthcare institutions should be made in collaboration with each other, as resistance patterns and disease outbreaks are likely to be similar in a defined region. The use of software like WHONET for surveillance reporting and antibiotic prescription should be utilized. The government healthcare authorities should take a proactive approach in this regards.

11. Use of information technology: In the present era, it is recommended that a conscious effort should be made to utilize the information technology tools for spreading awareness about the antibiotic stewardship program and to make it available easily, through personal digital assistants, hospital website, computers in the wards, etc. If the hospital has a hospital information system, there should be provision for inbuilt prompting of prescription.

12. In order to save the colossal amount of funds and gainfully utilise the resources, it is strongly recommended that there should be multidisciplinary approach to streamline the antibiotic prescribing practise with key pillars comprising of medical and antibiotic audits and surveillance studies supported by periodic training program.

\section{CONCLUSION}

Antimicrobial stewards are a prominent part of local and national efforts to contain and reverse antimicrobial resistance. A range of intervention options is available with varying levels of resources and can yield substantial improvements in morbidity, mortality, quality of care, and cost. The cost of delivering such programs is dwarfed by the benefits and provides an opportunity for hospital epidemiologists to garner support. This suggests that antimicrobial management programs belong to the rarefied group of truly cost saving quality improvement initiatives.

The rational use of drugs is of great importance in not only preventing the emergence of drug resistance but also to enable effective utilization of resources and bring down the cost of medical care. Antibiotics are an invaluable resource in our fight against infectious diseases which still today claim millions of lives. Irrational use of antibiotics is one of the reason cited for the increasing incidence of bacterial resistance. Antibiotic Stewardship program is one way, whereby we can ensure rational prescription of drugs.

Antimicrobial stewardship programs in hospitals seek to optimize antimicrobial prescribing in order to improve individual patient care as well as reduce hospital costs and slow the spread of antimicrobial resistance. With antimicrobial resistance on the rise worldwide and few new agents in development, antimicrobial stewardship programs are more important than ever in ensuring the continued efficacy of available antimicrobials. The design of antimicrobial management programs should be based on the best current understanding of the relationship between antimicrobial use and resistance. Such programs should be administered by multidisciplinary teams composed of infectious diseases physicians, clinical pharmacists, clinical microbiologists, and infection control practitioners and should be actively supported by hospital administrators. Strategies for changing antimicrobial prescribing behavior include education of prescribers regarding proper antimicrobial usage, creation of an antimicrobial formulary with restricted prescribing of targeted agents, and review of antimicrobial prescribing with feedback to prescribers. Clinical computer systems can aid in the implementation of each of these strategies, especially as expert systems able to provide patient-specific data and suggestions at the point of care. Antibiotic rotation strategies control the prescribing process by scheduled changes of antimicrobial classes used for empirical therapy. When instituting an antimicrobial 
stewardship program, a hospital should tailor its choice of strategies to its needs and available resources.

There have been a large number of studies carried out worldwide on various aspects of antibiotic policies, including their impact in saving healthcare costs. But there are hardly any studies conducted in India on antibiotic policies or the prescription patterns of antibiotics.

The present study carried out in a tertiary care government hospital retrospectively analyzed the case records of 100 patients admitted in the acute care wards of the hospital.

The study had also observed and discussed the criteria used for prescription of antibiotics, wherein it was seen that clinical criteria were used for antibiotic prescription of antibiotics in nine (9.\%) cases and, in the remaining 91 (91\%) cases, antibiotics were prescribed empirically. In these 91 cases, with antibiotics prescribed empirically, there was only four cases in which antibiotic was changed after culture and sensitivity tests.

The antibiotic stewardship program which is evolved after due deliberation and consideration over the resistance patterns, surveillance studies is aimed at providing scientific evidence based guidelines. It will help us to prescribe empirically on the various conditions mentioned in the stewardship program without fear of over or inappropriate prescription.

The study had also the analyzed as to whether associated instructions were mentioned clearly in the case records. The duration, dose, date of starting or stopping, other instructions regarding till what time the antibiotics were to be continued after discharge, legibility of prescription were inferred from the case records. The overall adherence to associated instructions including legibility of prescription was $52 \%$ with the highest incidence among case records belonging to surgical speciality. Keeping in view the growing incidence of legislation against doctors and introduction of right to information act in our country as well as patient safety point of view, it is highly imperative that this aspect of patient care is adhered to more stringently.

To conclude, this was a single worker study, and only carried out on inpatients of the hospital, the study did not take into account the outpatients patients and the knowledge and attitudes of prescribing clinicians and their apprehensions if any regarding the stewardship program. Since the acute wards considered in the study are the ones in which the chances of prescription of antibiotics is highest the study was confined to them. The study proposed to bring forth the enormous economic implications of inappropriate prescription of antibiotic not taking into account the economic burden of resistance created by it. The findings observed in the study are only the proverbial tip of the Iceberg, as it has not considered the gross over prescription of antibiotics being done over the counter and by incompetent and inadequately trained practitioners in the country.

It is essential considering the current scenario that the health administrative authorities enforce hospitals/ healthcare institutions to devise and implement antibiotic policies. Ideally, this stewardship program should be devised in consultation with other regional healthcare institutions and microbiology laboratory. The authorities should also take some steps towards curbing the inappropriate over the counter sale of antibiotics. Along with enforcing measures to prevent inappropriate prescription, the subject of antibiotic policies and issues related to it should be introduced in the curriculum of undergraduate medical students. The issues related to careful and appropriate prescription should be publicized with the help of health bulletins among the general public.

Considering the enormous implications of antibiotic resistance, it is necessary that we act in haste, lest our wonder drugs and magic bullets become ineffectual. Future systems promise greater integration and analysis of data, facilitated delivery of information to the clinician, and rapid and expert decision support that will optimize patient outcomes while minimizing antimicrobial resistance. They may also offer our best hope for avoiding an 'Antibiotic armageddon'.'

\section{REFERENCES}

1. Kunnin CM. Antibitic armageddon. Clin Infect Dis 1997; 25:240-241.

2. Mol PGM, Wieringa JE, Panday PVN, Gans ROB, Degener JE, Laseur M, Haaijer-Ruskamp FM. Improving compliance with hospital antibiotic guidelines: a time-146.

3. Calderdale and Huddersfield Hospital, NHS Trust, Antibiotic Stewardship Program, NHS Trust, 2006.

4. Royal Infirmary of Edinburgh, UK. Antimicrobial policies; 2006.

5. Guidelines for antimicrobial therapy, Christian Medical College, Vellore; 2005.

6. Wiffen PJ, White RTM. Encouraging good antimicrobial prescribing practice: A review of antibiotic prescribing policies used in the South East Region of England, British Medical Council, Public Health 2001;1:4.

7. Ozkurt Z, Erol S, et al. Changes in antibiotic use, cost and consumption after an antibiotic restriction Stewardship program imposed by infectious disease specialist. Japanese Journal of Infectious diseases 2005;58:337-343.

8. Fijn R, Chow MC. Multicentre evaluation of prescribing concurrence with anti-infective guidelines: epidemiological assessment of indicators, Pharmacoepidemiology and drug safety 2002;11(5):361-372.

9. Van Kasteren ME, Kullberg BJ, Adherence to local hospital guidelines for surgical antimicrobial prophylaxis: a multicentre audit in Dutch hospitals. Journal of Antimicrobial Chemotherapy, 2003;51(6):1389-1396.

10. Dey G. Study of antibiotic stewardship program at AIIMS, New Delhi; 2007. 